\title{
Avaliação de Epilepsia por Neuroimagem Estrutural. Protocolos
}

Henrique Carrete Júnior*

\author{
RESUMO \\ O objetivo principal deste artigo é fornecer os protocolos utilizados na UNIPETE/UNIFESP-EPM para o estudo de \\ ressonância magnética nas epilepsias focais. \\ Unitermos: Ressonância magnética, protocolos.
}

A ressonância magnética ( $\mathrm{RM})$, diferentemente da tomografia computadorizada (TC) ou de outro método de imagem, agrega um conjunto de técnicas que permitem avaliar diversos aspectos da estrutura, bioquímica e função do encéfalo, de modo não invasivo $^{1}$. Apesar de diversas causas de epilepsia poderem ser identificadas pela TC, algumas, como esclerose mesial temporal e pequenas áreas de displasia cortical, são claramente visibilizadas pela RM e geralmente não detectadas pela TC. As propriedades da RM, com maior contraste entre tecidos, capacidade de imagem multiplanar e ausência de artefatos produzidos pelo osso nas regiões de base do crânio, tão comuns na TC, fazem com que praticamente todas as lesões epileptogênicas sejam melhor identificadas e caracterizadas pela RM que $\mathrm{TC}^{2}$. Portanto, a RM é nos dias de hoje o método de escolha para investigação por imagem de pacientes com epilepsia, particularmente aqueles com crises refratárias. À TC resta um papel importante na detecção de calcificações.

Quando um exame de RM é realizado, diversas opções de técnicas podem ser escolhidas. Quando consideramos uma condição patológica específica, é importante que a técnica seja otimizada para identificar esta patologia, que é o objeto da investigação. Há muitas opções de tipos de seqüências a serem adquiridas, orientação e espessura de cortes, campo de visão, matriz, número de excitações, etc. Esses parâmetros devem ser definidos para cada entidade patológica que está para ser investigada, e não devemos aceitar que um exame básico ou de "rotina" seja adequado para a aquisição de imagens que permitam a identificação de todas estas importantes anormalidades.

Na realização de exame de RM devemos procurar obter imagens da melhor qualidade possível. Para tanto, queremos uma boa relação sinal-ruído, alta resolução espacial, cortes finos, elevado contraste entre diferentes tecidos e ausência de artefatos (por exemplo: movimentos). Também buscamos um tempo curto de aquisição e a possibilidade de reconstruirmos nossos dados em quaisquer planos.

O significado de otimização de um exame pode ser variado para diferentes especialistas envolvidos nesta investigação (neurologista, neurocirurgião, neurorradiologista etc.). Para o neurologista, otimização pode significar fazer o diagnóstico com o máximo de sensibilidade e especificidade possíveis, independentemente do tempo de duração do exame. Para o neurorradiologista que realiza a RM, otimização pode significar produzir imagens que permitam que o diagnóstico seja feito com o máximo de sensibilidade e especificidade, dentro de um tempo de exame determinado. Nesse momento, convém salientar que a produção de "belas" imagens pode não ter valor

Neurorradiologista, Departamento de Radiologia da UNIFESP - Escola Paulista de Medicina. 
algum se não houver contraste entre a região patológica e o tecido normal.

Portanto, os protocolos de aquisição de imagens por RM devem ser dirigidos de acordo com a suspeita clínica. E para tal, fica entendido como sendo fundamental a sintonia entre neuroclínicos, neurocirurgiões e neurorradiologistas. Cabe ao neurorradiologista, que protocola os exames, conhecer os substratos epileptogênicos e a ele deve ser oferecido o máximo de informações clínicas antes de se iniciar um exame.

A seguir, citamos as principais condições patológicas causadoras de epilepsia e a forma como estudamos esses pacientes pela RM.

\section{Esclerose mesial temporal}

O conhecimento da anatomia do lobo temporal medial é um pré-requisito fundamental para o reconhecimento e interpretação dos achados de imagem por RM na esclerose hipocampal ${ }^{3-7}$ (Figuras 1 e 2a, b, c e d). O hipocampo e a amígdala são melhor estudados em cortes coronais oblíquos, perpendiculares ao eixo longo do hipocampo, obtidos a partir de programação em corte sagital (Figura 3). Neste plano realizamos as seqüências FLAIR (fluid attenuated inversion recovery) e IR (inversion recovery), com

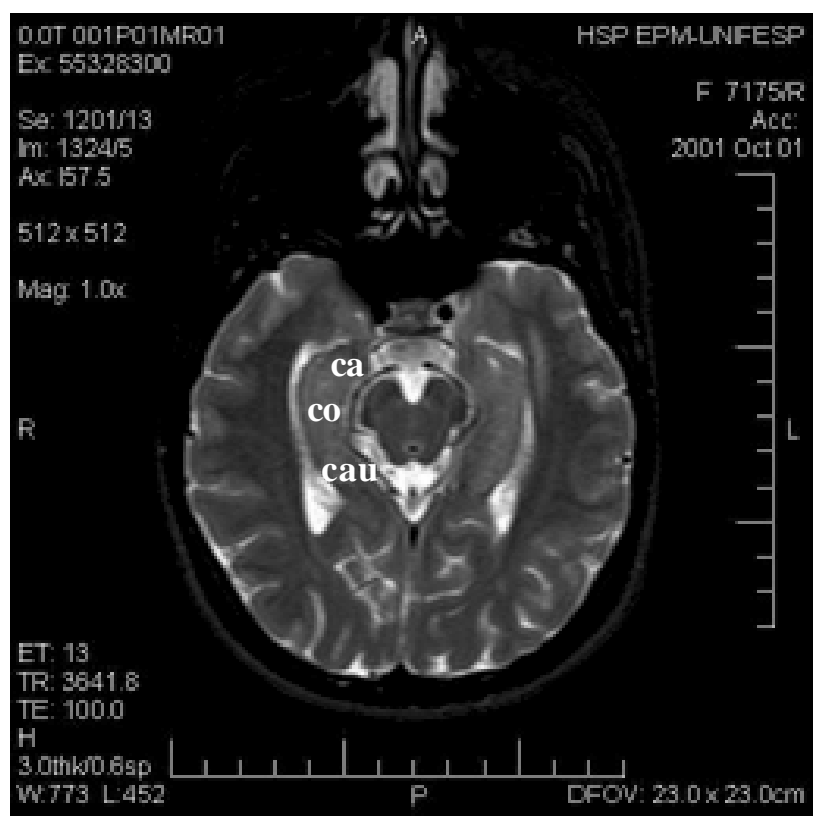

Figura 1 Seqüência turbo spin-echo ponderada em T2 axial paralela ao eixo longo do hipocampo, com corte de $3 \mathrm{~mm}$ de espessura. Observar neste tipo de plano a situação medial do hipocampo no lobo temporal e sua relação com o corno temporal do ventrículo lateral. Podemos identificar as três porções do hipocampo: cabeça, corpo e cauda. cortes finos. É necessário salientar a importância do posicionamento do paciente na mesa do equipamento e na orientação dos cortes, devendo-se evitar assimetrias artefatuais entre os dois hemisférios (Figura 2). Tanto o hipocampo como a amígdala são isointensos à substância cinzenta cortical em todas as sequiências de pulso de RM. O hipocampo, no entanto, pode ser ligeiramente hiperintenso à substância cinzenta na seqüência FLAIR devido à supressão incompleta do LCR 8 (Figura 4).

Para a análise das dimensões e arquitetura hipocampal, as seqüências IR são muito eficientes (Figuras 5 e 6). As alterações de sinal do hipocampo são bem determinadas pelas seqüências convencionais ou turbo spin-echo (fast spin-echo) ponderadas em T2, ou pela seqüência FLAIR (Figura 7), que permite ainda melhor contraste $\mathrm{T} 2$ para detecção de alteração de sinal ${ }^{9}$. Também são importantes as sequiências convencionais ou turbo spin-echo axiais para detectar anormalidades no restante do encéfalo.

\section{Anomalias do desenvolvimento cortical}

São malformações relacionadas à proliferação neuronal e glial anormais, migração neuronal anormal, ou organização cortical anormal. Essas podem ser focais, multifocais, ou generalizadas. Sequiências axiais ou coronais ponderadas em T2 e FLAIR são úteis para determinar alterações de sinal que, nos casos das displasias corticais, freqüentemente mostram áreas hiperintensas corticais e subcorticais. No entanto, algumas vezes nessa patologia, não há a alteração de sinal, ou seja, hiperintensidade em T2, havendo apenas espessamento cortical ou indefinição da junção córtico-subcortical. As seqüências IR são úteis nesses casos, por permitirem alto contraste entre substância cinzenta e branca (Figura 8). Também são úteis as aquisições volumétricas com cortes finos, que têm alta resolução espacial, e por permitirem exploração posterior em estação de trabalho, quando pesquisamos determinada região suspeita em diferentes planos, inclusive com reconstruções multiplanares (MPR) curvilíneas (Figura 9). O uso do contaste paramagnético não costuma ser útil na investigação dessas malformações, a não ser quando haja dúvida diagnóstica e se queira diferenciar de lesão de outra natureza, como por exemplo, de neoplasia glial.

\section{Neoplasias}

A RM é altamente sensível na detecção de neoplasias ${ }^{10}$. O principal objetivo da imagem é 

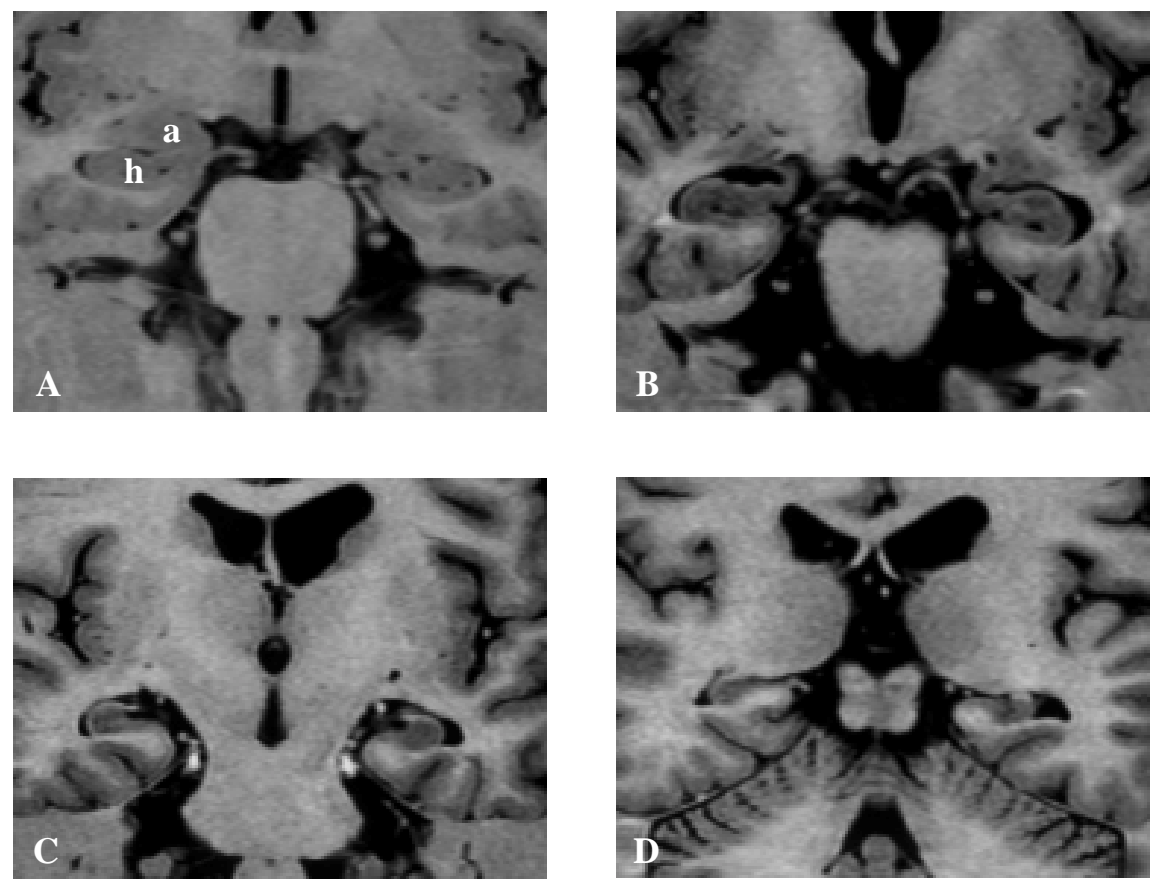

Figura 2 Seqüência IR coronal oblíqua, perpendicular ao eixo longo do hipocampo, com corte de 3 mm de espessura. Em A e B, os cortes passam pela região da cabeça do hipocampo que pode ser reconhecida pela presença de algumas digitações. Em A, notamos a relação da cabeça do hipocampo com a amígdala, que tem situação superior e mais anterior. Em B, o corte passa pela região do corpo do hipocampo, que tem formato ovalado, e em C, o corte passa pela sua cauda. Observar a perfeita simetria entre os dois hemisférios decorrente de um bom planejamento, o que pode ser comprovado pela comparação entre as estruturas da orelha interna e média dos dois lados $(A$ e $B)$.

demonstrar a lesão epileptogênica e diferenciá-la de outras entidades patológicas, não sendo possível em muitas das vezes predizer o tipo histológico da neoplasia apenas pela RM. Um sinal importante das neoplasias é o efeito de massa, no entanto, este achado pode estar ausente em mais de 1/3 das neoplasias associadas com epilepsia ${ }^{11}$. Geralmente os tumores responsáveis por epilepsia são pequenos e bem localizados, com pouco ou nenhum edema perilesional. O uso de seqüências multiplanares convencionais ponderadas em $\mathrm{T} 1$, sem e com contraste paramagnético, e sequiências convencionais ou turbo spin-echo ponderadas em T2, e FLAIR, são geralmente suficientes para a avaliação de neoplasias (Figuras 10a e b). Podemos em certos casos utilizar ainda seqüências volumétricas com reconstruções multiplanares, que poderão ser de grande auxílio no planejamento cirúrgico. Podemos ainda utilizar seqüências gradiente-echo $\mathrm{T} 2 *$ para detecção de calcificação ou hemorragia associada à neoplasia.

\section{Malformações vasculares}

A RM também é altamente sensível na detecção de malformações vasculares, que estão entre as causas importantes de epilepsia ${ }^{10}$. Além de seqüências convencionais ponderadas em $\mathrm{T} 1$ e turbo spin-echo ponderadas em T2, e FLAIR, é sempre útil a utilização de sequiências gradiente-echo ponderadas em T2*, que permitem caracterizar depósitos de hemossiderina na forma de halo em cavernomas, muitas vezes visíveis apenas neste tipo de seqüência (Figura 11).

\section{Gliose}

Diversos processos patológicos podem evoluir para gliose cortical e esta ser a causadora de crises. Nesse grupo de doença destacam-se as doenças inflamatórias e cerebrovasculares, e as lesões pós-traumáticas. Nestes casos, a gliose se mostra na imagem por RM como área de sinal hiperintenso em T2 e FLAIR, geralmente associada à perda de volume ${ }^{10}$. Dentre as causas inflamatórias/infecciosas, temos no nosso meio a neurocisticercose como uma entidade importante. As imagens de RM devem ser obtidas por seqüências convencionais ponderadas em $\mathrm{T} 1$, sem e com contraste paramagnético, sequências turbo spin-echo ponderadas em T2, e FLAIR, e também seqüências gradiente-echo T2* quando se deseja melhorar a sensibilidade do exame para a detecção de calcificações. 


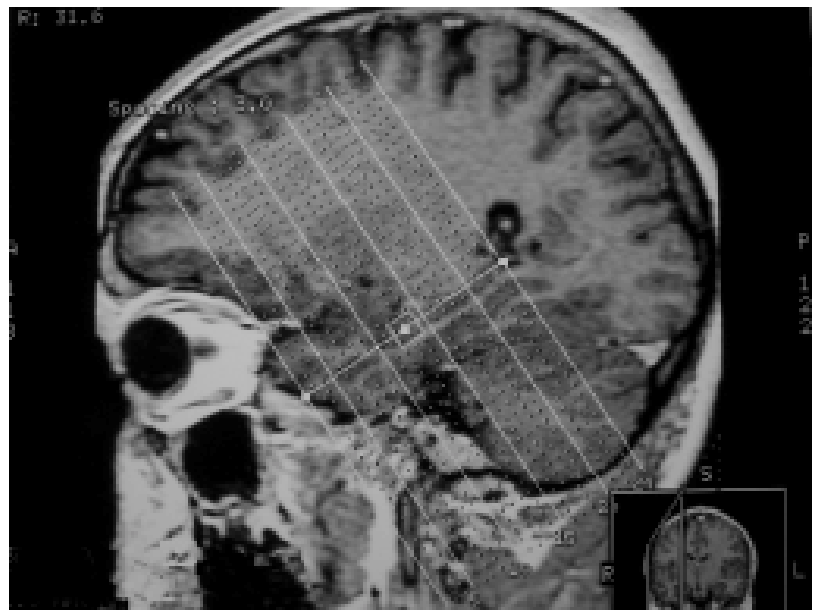

Figura 3 Sequiência localizadora ponderada em T1, sagital. Escolhemos a imagem que inclui um dos hipocampos para fazer a programação dos cortes coronais oblíquos das próximas seqüências. A orientação dos cortes deve ser perpendicular ao eixo longo do hipocampo.

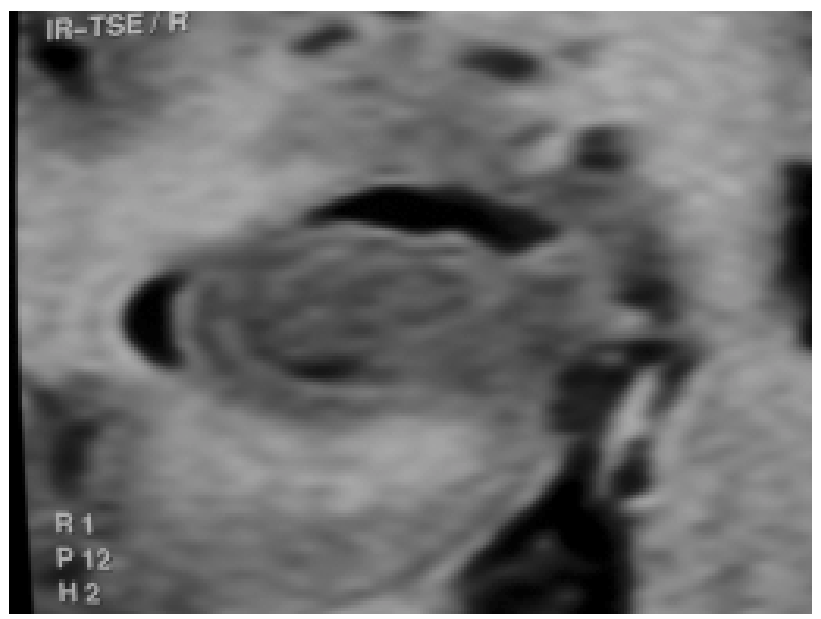

Figura 5 Seqüência IR coronal oblíqua, perpendicular ao eixo longo do hipocampo, com corte de $3 \mathrm{~mm}$ de espessura. Imagem magnificada da cabeça do hipocampo direito. Observar a sua arquitetura interna normal.

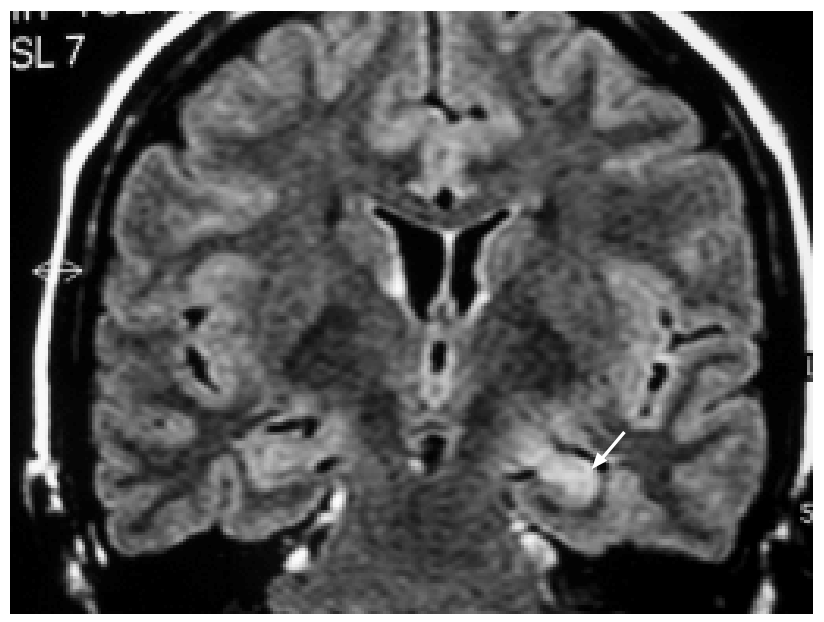

Figura 7 Seqüência FLAIR coronal oblíqua, com corte de $3 \mathrm{~mm}$ de espessura. O hipocampo esquerdo apresenta sinal hiperintenso na região do corpo. Comparar com o sinal do hipocampo direito normal. Esclerose hipocampal esquerda.

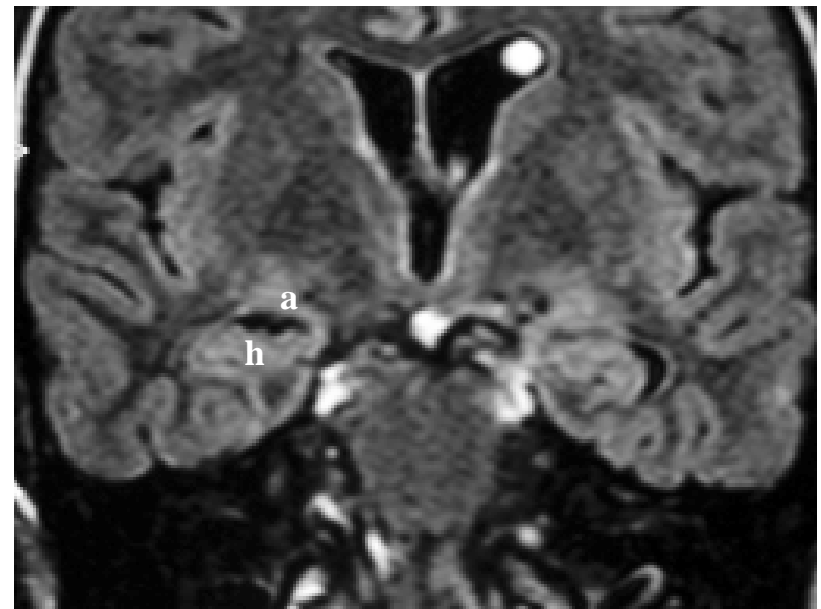

Figura 4 Seqüência FLAIR coronal oblíqua, com corte de $3 \mathrm{~mm}$ de espessura. Hipocampos e amígdalas de sinal normal, semelhante ao da substância cinzenta cortical, no entanto, os hipocampos têm sinal discretamente mais intenso, não devendo ser confundido com esclerose.

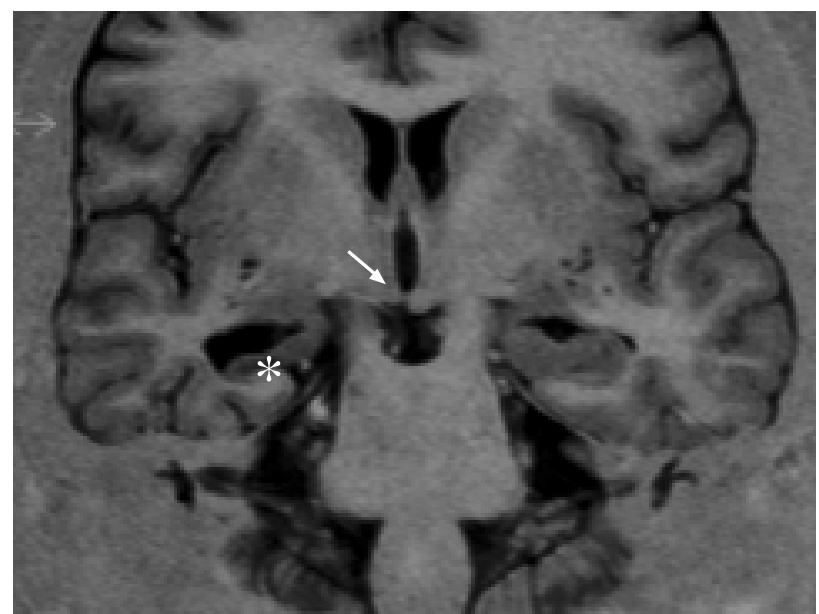

Figura 6 Sequiência IR coronal oblíqua, perpendicular ao eixo longo do hipocampo, com corte de $3 \mathrm{~mm}$ de espessura. $O$ hipocampo direito tem dimensões reduzidas em relação ao contralateral e perde a sua arquitetura normal. Notar ainda redução do volume do lobo temporal e do corpo mamilar do mesmo lado. Esclerose hipocampal direita.

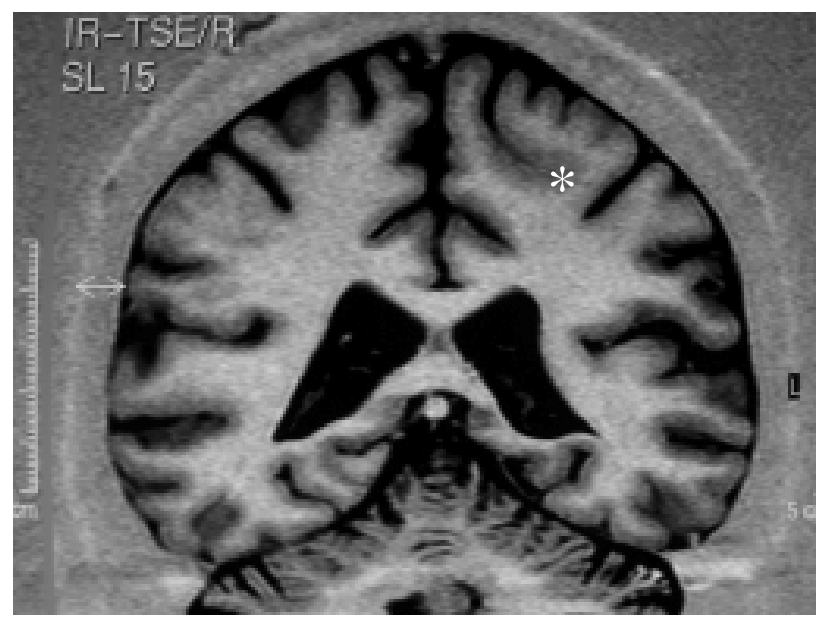

Figura 8 Seqüência IR coronal oblíqua, com corte de $3 \mathrm{~mm}$ de espessura. Espessamento cortical na região frontal pré-central esquerda. Displasia cortical frontal esquerda. 


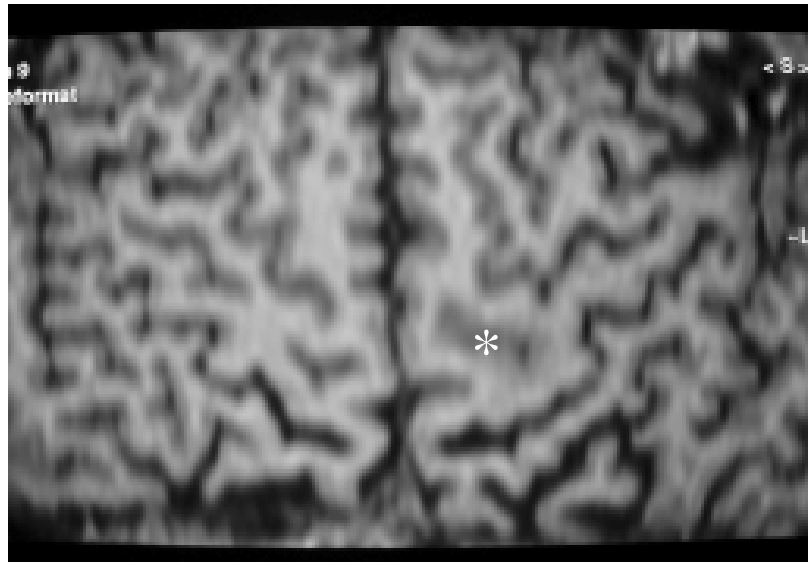

Figura 9 Seqüência volumétrica, gradiente-echo, com corte de $1,5 \mathrm{~mm}$ de espessura. Imagem reconstruída em estação de trabalho. Este tipo de reconstrução curvilínea possibilita identificar a área de espessamento cortical na região frontal pré-central esquerda com mais facilidade, utilizando-se de análise comparativa da anatomia dos giros e sulcos do hemisfério contralateral.

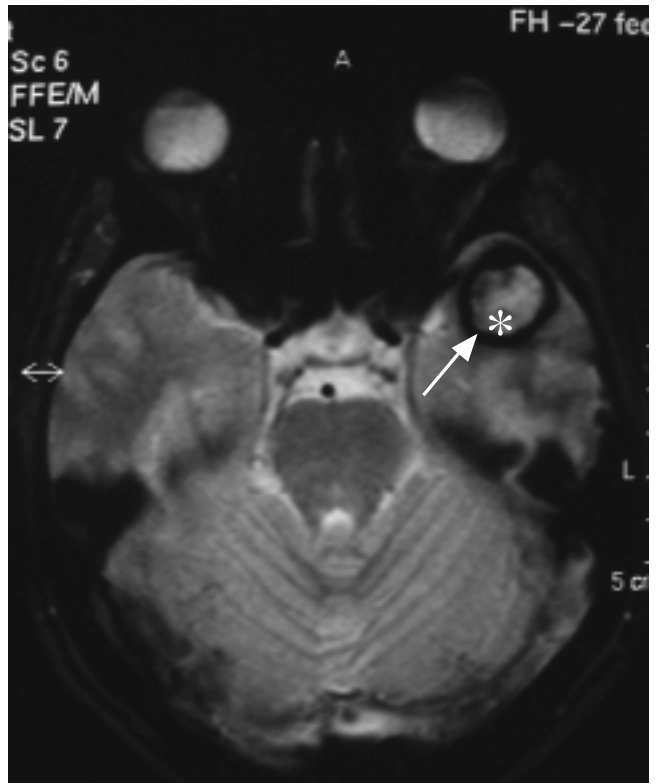

Figura 11 Seqüencia axial gradiente-echo T2*, com corte de $6 \mathrm{~mm}$ de espessura, no nível da fossa craniana média. No pólo temporal esquerdo notamos imagem com sinal hiperintenso central e halo de marcado sinal hipointenso (hemossiderina), característico de angioma cavernoso (cavernoma).

\section{SUMMARY}

Epilepsy evaluation by structural neuroimaging. Protocols

The main aim of this article is to discuss the UNIPETE-UNIFESP-EPM protocols which have been used in the evaluation of focal epilepsies.

\section{Keyw ords}

Magnetic resonance imaging, protocols.
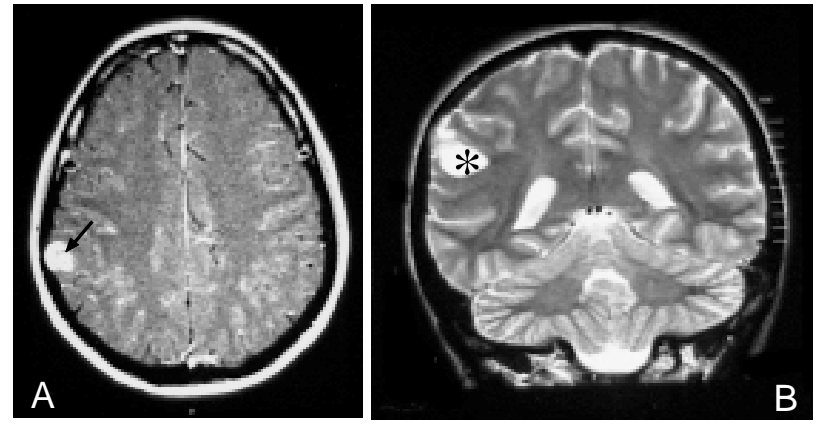

Figura 10 Seqüência axial spin-echo ponderada em T1, com corte de $5 \mathrm{~mm}$ de espessura, no nível dos centros semi-ovais, após injeção de contraste paramagnético; em A, observar o marcado realce desta lesão tumoral cortical parietal direita, que causa ainda remodelação do osso adjacente(seta). Seqüência coronal spin-echo ponderada em $T 2$, com corte de $5 \mathrm{~mm}$ de espessura, no nível dos átrios ventriculares; em $B$, observar a lesão cortical parietal esquerda com componente cístico(asterisco) e sem edema peri-lesional. Ganglioglioma.

\section{Referências}

1. Kuzniecky RI, Jackson GD. Magnetic resonance in epilepsy. New York, Raven Press Ltd, 1995, pp. 27-48.

2. Jack CR Jr. Magnetic resonance imaging - neuroimaging and anatomy. Neuroimaging Clinics of North America, 5:597-622, 1995.

3. Naidich TP, Daniels DL, Haughton VM et al. Hippocampal formation and related structures of the limbic lobe: anatomicMR correlation. Part I. Surface features and coronal sections. Radiology, 162:747-54, 1987.

4. Naidich TP, Daniels DL, Haughton VM et al. Hippocampal formation and related structures of the limbic lobe: anatomicMR correlation. Part II. Sagittal sections. Radiology, 162:75561, 1987.

5. Duvernoy HM. The human hippocampus. Functional anatomy, vascularization and serial sections with MRI, 2nd ed. Berlin: Springer-Verlag, 1998:1-72.

6. Bronen RA, Cheung G. MRI of the normal hippocampus. Magn Reson Imaging, 9:497-500, 1991.

7. Bronen RA. Hippocampal and limbic terminology. AJNR, 13:943-5, 1992.

8. Hirai T, Korogy Y, Yoshizumi K et al. Limbic lobe of the human brain: evaluation with turbo fluid-attenuated inversionrecovery MR imaging. Radiology, 215:470-5, 2000.

9. Jack CR Jr, Rydberg CH, Krecke KN et al. Mesial temporal sclerosis: diagnosis with fluid-attenuated inversion-recovery versus spin-echo MR imaging. Radiology, 199:367-73, 1996.

10. Bronen RA, Fulbright RK, Spencer DD et al. MR characteristics of neoplasms and vascular malformations associated with epilepsy. Magn Reson Imaging, 13:1153-62, 1995.

11. Atlas WS. Magnetic resonance imaging of the brain and spine, $3^{\text {rd }}$ ed. Philadelphia, Lippincott Williams \& Wilkins, 2002, pp.415-55.

\section{Endereço para correspondência:}

Escola Paulista de Medicina

Disciplina de Neurologia

Rua Botucatu, 740-m - Vila Clementino

CEP 04023-900 - São Paulo, SP

E-mail: egarzon@uol.com.br 